\title{
Can Fusion Proteins Be Considered a New Candidate for Tuberculosis Vaccine?
}

\author{
Sepehr Navid ${ }^{1}$ and Masoud Keikha (iD) ${ }^{1,}$ * \\ ${ }^{1}$ Department of Microbiology, School of Medicine, Isfahan Medical University, Isfahan, Iran \\ "Corresponding author: Department of Microbiology, School of Medicine, Isfahan Medical University, Isfahan, Iran. Tel: +98-9386836425, Email: masoud.keykha90@gmail.com.
}

Received 2018 May 29; Revised 2018 July 02; Accepted 2018 August 30.

\section{Dear Editor,}

Tuberculosis (TB) is one of the most important infectious diseases and the second leading cause of death by infectious agents after human immunodeficiency virus (HIV) (1). In 1982, all physicians in the worldwide believed that the disease would be controlled by the year 2000 and its discussion would be limited to case reports and medical literature, but this attitude lasted only for ten years where in 1993, TB was declared a global emergency by the World Health Organization (WHO) (2). Nowadays, with regard to the HIV epidemic growth around the world, as well as the emergence of multidrug-resistant TB (MDR-TB) and extensively drug-resistant tuberculosis (XDR-TB) strains, the eradication of tuberculosis is thought to be almost impossible $(1,2)$. According to the World Health Organization (WHO) in 2015, about 2 billion people worldwide are infected with Mycobacterium tuberculosis bacilli although they do not show any symptoms. In addition, 10.4 million new cases and 1.4 million TB-related deaths are estimated (1).

Currently, Mycobacterium bovis Bacillus CalmetteGuérin (BCG) is the only available vaccine against tuberculosis that has been used since 1921 to prevent tuberculosis. The BCG vaccine has only protective effects against meningitis and disseminated TB in children while it has minor protective effects and in some cases, no effects against tuberculosis in adults. Numerous clinical trial studies have shown that the protective effects of the BCG vaccine against pulmonary tuberculosis (PTB) range from 0 to $80 \%$ (3). In addition, the BCG vaccine does not prevent the reactivation of latent $\mathrm{TB}$. Therefore, it is imperative to develop efficient vaccines against TB in all forms, in particular, latent tuberculosis (4).

It is clear that vaccination is one of the most important protection and immunization strategies against infectious diseases, especially tuberculosis. In this regard, various tuberculosis vaccines such as DNA vaccines, viral vector vaccines, subunit vaccines, live-attenuated M. tuberculosis vac- cines, recombinant BCG vaccines, and some $M$. tb antigens have been proposed, among which subunit vaccines are of particular importance.

The most important principle in vaccine design is the correct recognition of immunogenic antigen and its presentation to the target system. In this context, adjuvants are very useful because they enhance antigen uptake and presentation of antigen-derived peptides by major histocompatibility complex (MHC) class II on antigenpresenting cells (APCs). Based on clinical studies, it is suggested selecting the early antigens of $M$. $t b$ during the proliferative phase and the latent phase $(4,5)$. The most important early antigens include ESAT-6 (6-kDa early-secreted antigen target), CFP-10 (10-kDa culture filtrate protein), peptide 190 - 198 of MPT64, and Ag85B. In addition, HspX protein (16-kDa $\alpha$-crystallin) and DosR (dormancy survival regulon) are the most important and effective antigens of $M$. $t b$ during the latent phase of the infection, which can induce proper immune responses in animal and human hosts $(6,7)$.

The immune system is based on two major classes: innate and acquired immunity. An effective immune response is the consequence of the interaction between these two classes. In addition, the acquired immunity consists of humoral and cell-mediated immunity, and the synergy between them is essential for events like affinity maturation, antibody class switching, memory cells formation, IFN- $\gamma$ production, $\mathrm{T}$ follicular helper (Tfh) cells formation, etc. (8). In this regard, it has been suggested that protein fusion containing primary and latent phase antigens attach to the FC region of immunoglobulin $\mathrm{G}(\operatorname{IgG})$, which can lead to the longevity of antigen-presenting, facilitation of antigen uptake by APCs (dendritic cells, macrophages, and B lymphocytes), enhanced possibility of cross-priming by dendritic cell (DC), increased solubility of antigens, stability and antigen protection from hydrolysis, correct folding of the antigens (due to the flexible hinge region), easy detection by staphylococcal protein A or polyhistidine-tag 
(6xHis tag), and easy purification of fusion protein $(9,10)$. Typically, antigen-derived peptides are expressed by MHC II on DCs, which can stimulate $\mathrm{TH}_{1}$, activate macrophages containing $M$. $t b$ by IFN- $\gamma$ production, and eventually eliminate bacteria within the phagolysosome by producing nitric oxide. Moreover, after antigen uptake during cross-priming by DC and antigen processing, antigen-derived peptides are presented to Cytotoxic T lymphocytes (CTLs) by MHC I, ultimately activating CTLS and eliminating the reservoirs of infection (10).

The important steps in fusion protein expression are choosing a suitable cloning vector, Codon optimization, and selecting an appropriate host. According to the literature, pUC57 is one of the best vectors, which contains multiple sites for restriction enzymes and it can carry a DNA fragment up to 300,000 nucleotides long. In addition, Pichia pastoris is one of the best systems for the expression of synthetic peptides compared to similar organisms because of its high growth rate, high proliferation capacity on the routine laboratory media, glycosylation, and posttranslational modifications.

The fusion peptides have been evaluated as one of the most important tuberculosis vaccines. There are 15 candidate vaccines (construction synthetic fusion peptide), which are being evaluated in clinical trials. Of these, H56 and ID93 that lead to an efficient immune response are of BCG vaccine boosters. These candidate vaccines are in phase I of clinical trials while Ag85B and ESAT-6 fusion peptide have entered phase IIa of clinical trials (11). According to the satisfactory results of studies on eukaryotic cell categories, animal models (such as mice, guinea pigs, goats, and monkeys), and clinical trials, there is a strong hope that the synthetic fusion proteins will be developed by the researchers and change to $M$. tb vaccines with higher potentials in comparison with the present vaccine, Mycobacterium bovis Bacille Calmette-Guérin (BCG vaccine) $(6,11-13)$.

So far, multiple vaccines consisting of fusion protein constructs containing $M$. tb antigens have been synthesized and used in numerous laboratory animal studies and clinical trials, leading to satisfactory results $(12,14,15)$. Currently, fusion proteins are widely used in such studies, and there is almost a general belief in the acceptance of synthetic peptides as a vaccine for tuberculosis (TB). However, this strategy requires more evolution in clinical trials for final approval (16).

\section{References}

1. World Health Organization (WHO). Global tuberculosis report 2015. WHO; 2015.
2. Daniel TM. The history of tuberculosis. Respir Med. 2006;100(11):186270. doi: 10.1016/j.rmed.2006.08.006. [PubMed: 16949809].

3. Li W, Deng G, Li M, Zeng J, Zhao L, Liu X, et al. A recombinant adenovirus expressing CFP10, ESAT6, Ag85A and Ag85B of Mycobacterium tuberculosis elicits strong antigen-specific immune responses in mice. Mol Immunol. 2014;62(1):86-95. doi: 10.1016/j.molimm.2014.06.007. [PubMed: 24980867].

4. Soleimanpour S, Farsiani H, Mosavat A, Ghazvini K, Eydgahi MR, Sankian M, et al. APC targeting enhances immunogenicity of a novel multistage Fc-fusion tuberculosis vaccine in mice. Appl Microbiol Biotechnol. 2015;99(24):10467-80. doi: 10.1007/s00253-015-6952-z. [PubMed: 26373723].

5. Pepponi I, Diogo GR, Stylianou E, van Dolleweerd CJ, Drake PM, Paul MJ, et al. Plant-derived recombinant immune complexes as self-adjuvanting TB immunogens for mucosal boosting of BCG. Plant Biotechnol J. 2014;12(7):840-50. doi: 10.1111/pbi.12185. [PubMed: 24629003].

6. Brandt L, Oettinger T, Holm A, Andersen AB, Andersen P. Key epitopes on the ESAT-6 antigen recognized in mice during the recall of protective immunity to Mycobacterium tuberculosis. J Immunol. 1996;157(8):3527-33. [PubMed: 8871652].

7. Liu X, Peng J, Hu L, Luo Y, Niu H, Bai C, et al. A multistage mycobacterium tuberculosis subunit vaccine LT70 including latency antigen Rv2626c induces long-term protection against tuberculosis. Hum Vaccin Immunother. 2016;12(7):1670-7. doi: 10.1080/21645515.2016.1141159. [PubMed: 26901244]. [PubMed Central: PMC4964814].

8. McHeyzer-Williams M, Okitsu S, Wang N, McHeyzer-Williams L. Molecular programming of B cell memory. Nat Rev Immunol. 2011;12(1):2434. doi: 10.1038/nri3128. [PubMed: 22158414]. [PubMed Central: PMC3947622].

9. Qiao SW, Kobayashi K, Johansen FE, Sollid LM, Andersen JT, Milford E, et al. Dependence of antibody-mediated presentation of antigen on FcRn. Proc Natl Acad Sci U S A. 2008;105(27):9337-42. doi: 10.1073/pnas.0801717105. [PubMed: 18599440]. [PubMed Central: PMC2453734].

10. Levin D, Golding B, Strome SE, Sauna ZE. Fc fusion as a platform technology: potential for modulating immunogenicity. Trends Biotechnol. 2015;33(1):27-34. doi: 10.1016/j.tibtech.2014.11.001. [PubMed: 25488117].

11. Khademi F, Derakhshan M, Yousefi-Avarvand A, Tafaghodi M, Soleimanpour S. Multi-stage subunit vaccines against Mycobacterium tuberculosis: an alternative to the BCG vaccine or a BCG-prime boost? Expert Rev Vaccines. 2018;17(1):31-44. doi: 10.1080/14760584.2018.1406309. [PubMed: 29148853].

12. Mosavat A, Soleimanpour S, Farsiani H, Sadeghian H, Ghazvini $\mathrm{K}$, Sankian M, et al. Fused Mycobacterium tuberculosis multistage immunogens with an Fc-delivery system as a promising approach for the development of a tuberculosis vaccine. Infect Genet Evol.2016;39:163-72. doi:10.1016/j.meegid.2016.01.027. [PubMed: 26835592].

13. Rowland R, McShane H. Tuberculosis vaccines in clinical trials. Expert Rev Vaccines. 2011;10(5):645-58. doi: 10.1586/erv.11.28. [PubMed: 21604985]. [PubMed Central: PMC3409871].

14. Pitt JM, Blankley S, McShane H, O'Garra A. Vaccination against tuberculosis: how can we better BCG? Microb Pathog. 2013;58:2-16. doi: 10.1016/j.micpath.2012.12.002. [PubMed: 23257069].

15. Langermans JA, Doherty TM, Vervenne RA, van der Laan T, Lyashchenko K, Greenwald R, et al. Protection of macaques against Mycobacterium tuberculosis infection by a subunit vaccine based on a fusion protein of antigen 85B and ESAT-6. Vaccine. 2005;23(21):274050. doi: 10.1016/j.vaccine.2004.11.051. [PubMed: 15780721].

16. Doherty TM, Andersen P. Vaccines for tuberculosis: novel concepts and recent progress. Clin Microbiol Rev. 2005;18(4):687-702. doi: 10.1128/CMR.18.4.687-702.2005. [PubMed: 16223953]. [PubMed Central: PMC1265910]. 